\title{
Educación e inmigración en Cataluña: un estado de la cuestión ${ }^{1}$
}

\author{
Diana López Falcón \\ Centre d'Estudis Demogràfics \\ dlopez@ced.uab.es
}

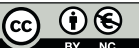

Recepción: noviembre de 2010

Aceptación: noviembre de 2010

\begin{abstract}
Resumen
Desde el comienzo del siglo xxi, Cataluña ha consolidado su posición como centro de atracción de población extranjera en el sur de Europa. Así, a 1 de enero de 2010 y de acuerdo con los datos preliminares del padrón continuo, la población de nacionalidad extranjera en Cataluña representaba un total de 1.193 .283 personas — un 15,9 por ciento de la población total. La entrada masiva de población extranjera en el territorio y el creciente debate respecto a la calidad de la educación han centrado el discurso en los mecanismos de integración y distribución en el sistema escolar de los niños y jóvenes de origen extranjero. El objetivo del presente trabajo es el de llevar a cabo una primera aproximación a la literatura sobre educación e inmigración en Cataluña, diferenciándola según tres temas principales: incorporación en el sistema educativo y rendimiento escolar; segregación escolar, y, finalmente, trayectorias educativas y estudios longitudinales.
\end{abstract}

Palabras clave: educación; inmigración; segregación escolar; trayectoria educativa; rendimiento escolar.

\section{Resum. Educació i immigració a Catalunya: un estat de la qüestió}

Des del començament del segle xxI, Catalunya ha consolidat la seva posició com a centre d'atracció de població estrangera del sud d'Europa. Així, a 1 de gener de 2010 i d'acord amb les dades preliminars del padró continu, la població de nacionalitat estrangera a Catalunya representava un total d'1.193.283 persones — un 15,9 per cent de la població total. L'entrada massiva de població estrangera en el territori i el creixent debat respecte a la qualitat de l'educació han centrat el discurs en els mecanismes d'integració i distribució en el sistema escolar dels nens i joves d'origen estranger. L'objectiu del present treball és el de dur a terme una primera aproximació a la literatura sobre educació i immigració a Catalunya, segons tres temes principals de recerca: incorporació en el sistema educatiu i rendiment escolar; segregació escolar, i, finalment, trajectòries educatives i estudis longitudinals.

Paraules clau: educació; segregació; població estrangera; trajectòria educativa; rendiment escolar.

1. Este texto se inscribe dentro del proyecto de I+D Comportamientos sociodemográficos diferenciales e integración social de la población inmigrada y de sus descendientes en España (CSO2008-04778/SOCI), dirigido por el Dr. Andreu Domingo y financiado por el Ministerio de Ciencia e Innovación, mediante el Plan Nacional de I+D+I 2008-2011. 
Résumé. Education et immigration en Catalogne: un état de la question

Depuis le début du Xxi siècle, la Catalogne a renforcé sa position de focus d'attraction des populations étrangères dans le sud de l'Europe. Ainsi, au premier janvier 2010, et selon les données préliminaires du recensement continu (padrón continuo), la population de nationalité étrangère en Catalogne représentait un total de 1.193 .283 personnes, soit $15,9 \%$ de la population totale. L'entrée massive de population étrangère sur le territoire et le débat croissant sur la qualité de l'éducation ont centré le discours sur les mécanismes d'intégration et de répartition au sein du système scolaire des élèves d'origine étrangère. L'objectif du présent travail consiste à établir une première approche de la littérature traitant de l'éducation et de l'immigration en Catalogne, au tour de trois thèmes principaux: l'incorporation dans le système éducatif et la performance scolaire; la ségrégation scolaire; finalement, les trajectoires éducatives et les études longitudinales.

Mots clé: éducation; immigration; ségrégation scolaire; trajectoire éducative; rendement scolaire.

\section{Abstract. Education and immigration in Catalonia: A state of the art}

From the beginning of the 21 st Century, Catalonia has consolidated its position as an international migration attraction node in southern Europe. As a result, at January 1st, $2010,15.9 \%$ of the Catalan population (1'193,283 persons) was of foreign origin. The massive foreign population in-flows and the growing debate on the quality of education have led to focus the attention on the mechanisms of integration and distribution of foreign origin students. The paper's aim is to present a first approach to the scientific literature on education and international migration in Catalonia on three main fields: access to education and academic performance; school segregation; and, finally, academic trajectories and longitudinal studies.

Key words: education; school segregation; foreign population; school trajectories; academic performance.

\begin{aligned} & \multicolumn{2}{c}{ Sumario } \\ & Introducción Trayectorias educativas y estudios \\ & $\begin{aligned} \text { Incorporación en el sistema educativo y } \\ \text { rendimiento escolar }\end{aligned}$ longitudinales \\ & Segregación escolar Referencias bibliográficas \end{aligned}

\section{Introducción}

A lo largo de las dos últimas décadas, España ha experimentado un cambio en su posición migratoria neta, puesto que se ha convertido en uno de los principales receptores de población de Europa. Cataluña se ha consolidado como uno de los principales receptores de flujos migratorios provenientes del extranjero en España, hasta alcanzar un 15,9\% de su población total - 1.193 .283 personas-, de acuerdo con los datos preliminares del padrón continuo a 1 de enero de 2010. Como era de esperar, el debate en torno a la integración social de la población de nacionalidad extranjera en España ha estado relacionado no sólo 
con el acceso al mercado laboral y los servicios, sino con la escolarización de la población más joven recién llegada.

Los primeros estudios sobre inmigración y educación en España aparecieron en torno a 1991, coincidiendo con el incremento de población extranjera en el territorio. Anteriormente, y en especial en el campo de la educación intercultural en España, los estudios se centraban principalmente en la escolarización del alumnado de etnia gitana, y 1992 fue el gran punto de inflexión hacia la inmigración internacional (García Fernández, 2006). La presencia de niños y jóvenes de origen extranjero en la etapa de escolarización obligatoria incentivó los primeros estudios sobre escolarización de los hijos de inmigrantes marroquíes (Aparicio, 2001), pero en el caso específico de Cataluña, este campo de investigación es relativamente nuevo, a pesar de haber sido tratado con anterioridad en estudios realizados para el conjunto español.

El objetivo del presente trabajo es el de llevar a cabo una primera aproximación a la literatura sobre educación e inmigración para Cataluña desde diferentes campos de investigación científica. Cabe destacar que hemos considerado no sólo aquella literatura enfocada en el Principado, sino también en los estudios que lo han incluido como parte de una muestra para el total del país. Dada la diversidad de enfoques que han tratado este tema, hemos limitado nuestro análisis a tres grandes temas: incorporación en el sistema educativo y rendimiento escolar; segregación escolar, y, finalmente, trayectorias educativas y estudios longitudinales.

\section{Incorporación en el sistema educativo y rendimiento escolar}

Una de las mayores preocupaciones de la sociedad desde el inicio del reciente boom migratorio ha sido sin duda el acceso a la educación. Por una parte, el debate se había centrado en la distribución de los alumnos en los centros escolares — en ocasiones, focalizándose en los de titularidad pública - y cómo su incorporación afecta al rendimiento escolar. Dado que la mayoría de los flujos que han arribado a Cataluña provienen en su mayoría de países o regiones catalogadas como «subdesarrolladas» o pobres, como lo son América Latina, África o Europa del Este, a menudo se considera que su incorporación a los centros escolares representará un perjuicio para el alumnado español. Cuando éste, además, se concentra en determinados centros escolares, tiende a considerarse en la agenda política y, en general, en el debate público, como un «problema» de primer orden (Alegre, 2005; Alegre et al., 2005). El objetivo del presente apartado es el de exponer los trabajos en materia de integración al sistema educativo y aquellos que hacen referencia al rendimiento educativo del alumnado en Cataluña desde diferentes perspectivas.

En el campo de la sociología, Aja et al. (1999) analizan la regulación del acceso educativo para los niños y jóvenes de origen extranjero en España, así como el proceso de integración, la diversidad de los estudiantes y la percepción de la sociedad española sobre el alumnado de origen extranjero. Los estudios de Alegre $(2005,2006)$ analizan la situación del alumnado extranjero en Cataluña 
desde el punto de vista de los principales actores afectados (profesores, padres de familia, alumnos). El autor incluye en su estudio al alumnado de origen extranjero con necesidades educativas específicas (NEE) por razones de incorporación tardía al ciclo escolar. En ese sentido y respecto al alumnado nativo, las necesidades educativas del alumnado inmigrante están relacionadas con la edad de llegada e inicio de la escolarización; el conocimiento o no de la lengua autóctona, y la trayectoria educativa y el nivel de aprendizaje desarrollado en el país de origen. El monográfico del CIDE (2005) presenta un compendio del marco legal y los programas autonómicos destinados a la atención del alumnado de origen extranjero de nueva incorporación, así como un sumario sobre la investigación en materia de educación de los últimos veinte años. Por su parte, Siqués (2008) plantea los diferentes programas de acogida lingüística, así como la evaluación de las aulas de acogida de educación primaria en Cataluña para el curso escolar 2006-2007.

Desde el campo de la pedagogía, los estudios de Garreta (2006), Essomba (2002), Santamaría $(1994,2002)$ y Carbonell (1998) analizan las relaciones de los estudiantes de origen extranjero dentro de las aulas y los procesos de integración, pero en ningún caso tratan de estudios longitudinales del ciclo formativo y/o profesional de los alumnos entrevistados. Bajo esta misma línea, los estudios de Essomba $(1998,2003)$ y Ferrer, Essomba y Bonal (2005) llevan a cabo un análisis de la situación actual, pero lo hacen desde el punto de vista de profesores y educadores respecto a los retos que presenta el alumnado recién llegado y la efectividad de las políticas autonómicas. Desde el campo de la antropología y la etnografía del alumnado de origen extranjero, Carrasco (2003) incide en el debate actual sobre la multiculturalidad educativa de los estudiantes de origen extranjero y su relación con los de etnia gitana.

Uno de los primeros estudios sobre los retos de la escuela y el diseño de las políticas públicas para la incorporación de alumnado de origen extranjero en Cataluña tras las migraciones más recientes es el de Palaudàrias (2002). En él, el autor examina las diferentes respuestas desde el sistema educativo, como el Programa de Educación Compensatoria para niños y jóvenes de origen extranjero, que, en muchos casos, son categorizados como inmigrantes, cuando, en realidad, nacieron en territorio español o hace tiempo que viven aquí. Alegre (2008) hace una profunda revisión de la problemática frecuentemente citada en la literatura como resultado de la integración en el sistema escolar de las hijas e hijos de los inmigrantes, así como de los autores más relevantes, tanto a nivel teórico como aplicado. Por otra parte, el autor estudia los diversos sistemas de acceso escolar, poniendo frente a frente los retos que presenta la integración de alumnado proveniente de otros sistemas educativos y el desarrollo de políticas educativas.

En cuanto al rendimiento escolar, Sánchez Hugalde (2007) y el Colectivo Ioé (2003) coinciden respecto a la correlación negativa entre el número de estudiantes de origen extranjero matriculado en los centros y el rendimiento escolar global. Sin embargo, en términos de rendimiento, el efecto negativo de la concentración afecta más al alumnado extranjero que al nativo. En cualquier 
caso, los resultados escolares no son los mismos para todas las nacionalidades, ya que se encuentran diferencias significativas cuando se incluye el país de origen en el estudio.

Recientemente, los estudios de Zinovyeva et al. (2009) para España y Ferrer et al. (2008) para Cataluña llevan a cabo una aproximación a las diferencias del rendimiento educativo entre inmigrantes y nativos tomando como base los datos del Programa Internacional de Evaluación de Estudiantes (PISA). El alumnado inmigrante presenta un rendimiento inferior que los nativos, cuestión que los autores justifican dadas las diferencias observables en las características socioeconómicas de los padres. Más allá, Zinovyeva et al. (2009) analizan las políticas que pueden disminuir la brecha educativa entre alumnado de origen extranjero y nativo, lo cual incidirá en su inserción laboral a largo plazo, que, a su vez, reducirá el impacto socioeconómico de esta desigualdad. Tal como mencionan los autores, aún son escasos los estudios sobre los determinantes de la brecha educativa entre el alumnado, siendo los existentes esencialmente descriptivos (Defensor del Pueblo, 2003; Ministerio de Educación y Ciencia, 2006).

\section{Segregación escolar}

La desigual distribución de la población extranjera en el territorio ha dado pie a dos de las principales líneas de investigación sobre población extranjera: la segregación espacial del conjunto de la población y la segregación escolar. De acuerdo con Massey y Denton (1988), la segregación escolar se refiere al grado con el que dos o más grupos asisten a la escuela de forma diferenciada en un área geográfica específica. En este sentido, la segregación escolar es en cierta manera un reflejo de la misma desigualdad urbana y la segregación urbana existente. No obstante, de acuerdo con autores como Schindler (2007) o Gramberg (1998), los niveles de segregación escolar son tradicionalmente más elevados que su contraparte residencial. Esto puede estar influenciado por la diferente composición por edades de la población extranjera y española, así como por la salida del alumnado nativo - especialmente cuando se cuenta con una red educativa doble, pública y privada- o entre escuelas vecinas con la misma titularidad (Valiente, 2008). Por ello, la segregación escolar es vista con preocupación, al resultar un obstáculo en el proceso de integración en la sociedad de acogida, puesto que impide el contacto de los alumnos de origen extranjero con los nacionales y dificulta así el establecimiento de las redes sociales entre ambos grupos. De la misma forma, la segregación residencial se podría considerar como la incubadora para la transmisión intergeneracional del capital social entre personas de un mismo origen - también conocido como capital étnico - y es por ello que la segregación del lugar de residencia y dentro de los centros escolares ha sido objeto de numerosas investigaciones relacionadas con el rendimiento escolar.

Dadas las limitaciones derivadas del acceso a fuentes de datos públicas, la segregación escolar ha sido analizada por diversos autores frecuentemente 
a escala local —comunidad autónoma, municipio, escuela...- y a menudo a través de la aplicación de encuestas ad hoc en una muestra específica de centros educativos. En el ámbito español, Aja et al. (1999) analizan la distribución espacial de los estudiantes, haciendo especial énfasis en la concentración «natural» $\mathrm{y}$ «artificial», tanto del alumnado de origen extranjero como del español, en función de la elección del centro educativo. En este mismo sentido, los autores consideran que la causa principal de la concentración artificial y, por tanto, de la guetización de algunos centros escolares de España son las leyes autonómicas, que no representan un entramado lo suficientemente robusto para evitar este tipo de incidencias. El Colectivo Ioé (2002) presenta un análisis descriptivo de la distribución y la composición del alumnado en los centros educativos mediante el estudio de los datos de las estadísticas de educación de España y su relación con los nacimientos de niños de origen extranjero. Por su parte, Alegre (2008) analiza los diferentes mecanismos de redistribución del alumnado inmigrante, que, como es de esperar, inciden directamente en la segregación acadèmica y presenta los tres modelos de zonificación escolar ${ }^{2}$ basados en el estudio de Benito y González (2007b). Si bien es cierto que originalmente estuvieron enfocados al impacto de los diferentes modelos de zonificación escolar sobre el nivel de equidad entre la composición social de las distintas escuelas en diferentes municipios catalanes, la clasificación que establecen a propósito de las diferentes tipologías de zonificación es generalizable al conjunto del territorio español (Alegre, 2008).

Para Cataluña, la relevancia del carácter territorial a escala local ha sido recientemente incorporada en los estudios de Benito y González (2007a), el Síndic de Greuges (2008) y Sánchez Hugalde (2007). Benito y González (2007a) analizan la segregación escolar y el efecto de las políticas escolares mediante la aplicación de encuestas en una muestra de municipios catalanes y su posterior contraste con los datos relativos a matriculación. Por su parte, el Síndic de Greuges (2008) estudia en profundidad el análisis de la segregación escolar desde el punto de vista territorial, teniendo en cuenta las políticas para combatir este fenómeno y la evidencia empírica aportada por las quejas presentadas ante esta institución. Específicamente, el estudio profundiza sobre la segregación escolar experimentada en el territorio gerundense, dado que es la zona con mayor segregación escolar de Cataluña. Finalmente, Sánchez Hugalde (2009) hace un análisis de la relación entre la segregación residencial y la escolar para el curso académico 2002-2003 para aquellos municipios con más de 20.000 habitantes y con al menos 10 habitantes de origen extranjero. A pesar de su diferente naturaleza, los estudios confirman el efecto adverso de la segregación de la población escolar de origen extranjero, no sólo sobre ella misma, sino también sobre el agregado en términos de rendimiento escolar e integración.

Alegre, Benito y González (2008) analizan la incidencia de las políticas de zonificación escolar sobre la segregación y la polarización de la red educativa

2. Se entienden las políticas de zonificación escolar como las políticas de preferencia de asignación de plaza a través de la delimitación de zonas de influencia en los centros docentes. 
en diez municipios. Entre los resultados encontrados, destacan que el modelo que asigna una única escuela a cada área de influencia incentiva la reproducción residencial en el entramado escolar, mientras que la asignación de varios centros educativos por zona favorecerá su disminución. A pesar de que - tal como los autores reconocen - la muestra de municipios empleada no permite su extrapolación a un nivel superior, las principales aportaciones del estudio son la combinación del análisis de la segregación escolar con el del contexto familiar (perfil socioeconómico y capital instructivo familiar) y, más específicamente, con el nivel de escolaridad de los padres, así como el análisis de los resultados de las políticas de acceso a la educación empleadas.

A modo de compendio, Carrasco (2008) revisa elementos clave en el debate sobre las mejores estrategias de escolarización del alumnado de origen extranjero para garantizar su posterior incorporación a la sociedad catalana en condiciones de igualdad, a través de la revisión de diversos estudios de índole nacional e internacional. Carrasco et al. (2009) presentan el estudio de las dinámicas formales e informales de separación del alumnado dentro de las escuelas. Tal como mencionan los autores, "parece necesario prestar atención no sólo a la segregación interescolar desde la perspectiva de los procesos sociales que inciden en las desigualdades educativas, sino también a las trayectorias disponibles para el alumnado dentro de las instituciones educativas para comprender los procesos y los efectos de la segregación intraescolar frente a los objetivos de la inclusión educativa» (Carrasco et al., 2009: 3). En este sentido, cabe destacar la forma en la que cambia el discurso de los padres dependiendo de la etapa escolar en la que están escolarizados sus hijos y la reproducción de las jerarquías socioculturales en la escuela.

\section{Trayectorias educativas y estudios longitudinales}

En el contexto internacional, la educación ha sido profundamente estudiada, no sólo como una herramienta de integración social, sino también como una conexión directa entre el proceso migratorio y el mercado laboral local. Tomando en cuenta la definición de capital humano en el sentido de Becker (1964), como la acumulación de habilidades y conocimientos que influirán en las ganancias futuras de los individuos, es de esperar que los modelos de inmigración que incluyan esta variable concluyan que, para stocks iniciales similares, las ganancias futuras sean las mismas entre inmigrantes y nativos. Por tanto, las inversiones en educación traerán consigo un incremento de los ingresos futuros debido a la influencia de ésta en la calidad del trabajo, lo cual beneficiará además al conjunto de la economía, dada su contribución al crecimiento económico.

Cuando el análisis se ha centrado en la población de nacionalidad extranjera, ha hecho especial incidencia en la relación entre el proceso de integración y el propio capital humano o étnico, valorando las diferencias entre nacionalidades y entre nativos e inmigrantes como indicadores del proceso de integración. $\mathrm{O}$, desde un punto de vista contrario, como forma de detectar filtros de exclu- 
sión y discriminación que podrían afectar de manera dispar a los diferentes colectivos por barreras formales o informales.

Dado que la educación desempeña un papel central en el proceso de acumulación de capital humano, un aspecto clave es en qué medida los logros educativos de los estudiantes hijos de inmigrantes difieren con respecto a los de los nativos, especialmente en lo que respecta a la inversión en educación postobligatoria (Aina et al., 2008). Sin embargo, la proporción de alumnado de origen extranjero decae significativamente en los niveles de educación postobligatoria y, en especial, en el universitario, por lo que podemos considerar que lo anterior se debe a su temprana incorporación al mercado laboral. A pesar de no tratarse de estudios específicos sobre trayectorias o transiciones educativas, podemos encontrar de forma disociada aproximaciones desde el campo del análisis económico o la sociología del trabajo referentes a la integración laboral de la población extranjera (Fernández Anguita (dir.), 2007; Pajares et al., 2004) y las características sociodemográficas (entre ellas, el nivel de instrucción) según la actividad de la población. Por otra parte, Alonso y Sosvilla-Rivero (2006) encontraron que los bajos niveles de educación alcanzados en el país pueden explicarse por el porcentaje de población trabajadora por cohorte. Los autores llevaron a cabo una estimación del capital humano en España a partir de datos de la Encuesta de Población Activa (EPA). En algunas de las cohortes analizadas, la población con un nivel de educación inferior al obligatorio superó el 60\% y mostraba un rápido abandono escolar, dado el alto costo de oportunidad de seguir estudiando.

Debido al reciente proceso de asentamiento de la población inmigrante, de momento no ha sido posible verificar si se ha producido una movilidad ascendente, tanto entre los individuos como entre sus descendientes. Una de las principales limitaciones que enfrenta el estudio de la población escolarizada es sin duda la ausencia de una base de datos detallada a nivel nacional de acceso público. En este sentido, Carrasco (2003) hace énfasis en las dificultades que representan las bases de datos disponibles debido al registro erróneo de los estudiantes en los centros educativos, así como la variación en las series temporales dependiendo de la normativa de educación vigente a lo largo del tiempo. Carrasco (2008) y Cebolla (2008) mencionan la falta de recursos que permitan estimar la desventaja educativa y el resultado de las políticas aplicadas. Tal como expone Carrasco (2008), los datos disponibles sobre inmigración y educación no permiten evaluar los efectos de las políticas educativas ni de las prácticas escolares aplicadas a las diferentes cohortes, ni tan sólo es posible construir, por ejemplo, indicadores sobre la tasa de éxito escolar del alumnado extranjero ni profundizar sobre las desigualdades que se observan (Carrasco, 2008).

En una aproximación al estudio del abandono, el fracaso escolar y la transición entre la etapa obligatoria y la postobligatoria, Serra y Palaudàrias (2007) presentan un análisis a partir de datos del Departamento de Educación y una muestra propia de dieciocho centros educativos de secundaria. Los autores ponen de manifiesto lo que consideran un déficit fundamental en el seguimiento de las trayectorias académicas de los alumnos. En la medida que la 
capacidad de conocer la realidad sea limitada, podemos suponer que también lo serán las posibilidades de detectar problemas y, por consiguiente, la atención que se pueda prestar al respecto.

Aproximaciones longitudinales mediante encuestas ad hoc, como las propuestas por Portes y Zhou (1993), Portes y Rumbaut (2003) o Borjas (1999) para los Estados Unidos, o la de Gang y Zimmermann (2000) para Alemania, hasta el momento no han sido viables. En ellas, se destaca la dimensión positiva de las redes sociales o étnicas en el avance educativo de los niños y jóvenes de origen extranjero. Una de las primeras aproximaciones de esta índole son los estudios de Aparicio (2007) y Aparicio y Tornos (2006) para las nacionalidades con una trayectoria migratoria más antigua en las ciudades de Madrid y Barcelona. Los autores analizan el acceso a la educación y al mercado de trabajo de las llamadas generación 1,5 y segunda generación de inmigrantes de origen marroquí, dominicano y peruano mediante la aplicación de encuestas específicas. Buscando verificar la posible existencia de movilidad social ascendente, la autora encontró que, a pesar de experimentar un ligero beneficio respecto a la generación de sus padres, los logros y los resultados de la segunda generación aún distan de los nativos. Sin embargo, el resultado no es el mismo para los tres grupos analizados, puesto que beneficia principalmente a los peruanos. De acuerdo con los autores, lo anterior puede deberse a diferencias en la estructura familiar y en las redes sociales de cada una de las nacionalidades.

Recientemente, Portes et al. (2009) presentan los resultados de la primera fase de un estudio longitudinal de la segunda generación en España. A pesar de incluir las mismas ciudades en el análisis, a diferencia de los estudios de Aparicio (2007) y Aparicio y Tornos (2006), la muestra considerada en esta investigación se centró en estudiantes de doce a diecisiete años de más de sesenta nacionalidades y escolarizados en centros de titularidad tanto pública como privada. Durante esta primera etapa de investigación, se pretendía conocer las aspiraciones y las expectativas educativas y laborales dependiendo de las características de los alumnos y sus familias, que en algunos casos no se han visto cubiertas con la realidad educativa.

\section{Conclusiones}

El incremento en la demanda de escolarización, especialmente en la etapa obligatoria —entre los seis y los dieciséis años de edad-, ha generado no sólo desigualdades en el acceso a la educación, sino que ha incidido directamente en la segregación escolar, tanto de la población extranjera como de la nativa. Sin embargo, a pesar de que este incremento en la demanda ha centrado la investigación en la inmigración internacional, otro de los componentes de su crecimiento es el de la recuperación en la fecundidad de la población nativa de mediados de los años noventa. En términos generales, es el incremento del alumnado el que ha puesto en evidencia las carencias y los puntos débiles del sistema educativo, que, en su mayoría, son independientes del lugar de origen de la población escolarizada. 
Es por ello que, para entender la problemática del sistema educativo, no basta con estudiar a la población de origen extranjero recientemente escolarizada. Es necesario hacer una reflexión sobre la incidencia de la política educativa y, sin duda alguna, extender los mecanismos de intervención también a la población nativa, especialmente en lo que se refiere a la lucha contra el fraude en el acceso a determinados centros educativos. En este sentido, es sin duda loable el esfuerzo de los investigadores por llevar a cabo estudios cuantitativos a pesar de la limitación de los datos disponibles, así como la aplicación de encuestas específicas para el estudio de este fenómeno.

Por otra parte, en el estudio de la relación entre educación e inmigración, no sólo es importante el analizar hasta qué punto se verán afectados los niveles de aprovechamiento escolar. De acuerdo con los últimos resultados del programa PISA, la apremiante necesidad de inversión en educación no debe ir sólo encaminada al aprovechamiento escolar, sino también a evitar el abandono escolar a nivel secundario. Tal como exponen Zinovyeva et al. (2009), la brecha educativa entre nativos e inmigrantes no sólo es la causante de la segregación laboral de las segundas generaciones de inmigrantes, sino también de su marginalidad socioeconómica, origen a su vez de conflictos sociales. No sólo es importante garantizar el derecho a la educación en la etapa obligatoria, sino también permitir la continuidad de los estudios en las etapas posteriores y garantizar la inserción social y laboral de la población independientemente del origen.

\section{Referencias bibliográficas}

Aina, C.; Casalone, G. y Ghinetti, P. (2008). Internal geograpnical mobility and educational outcomes: An analysis for an Italian province. SEMEQ DepartmentFaculty of Economics-University of Eastern Piedmont.

Aja, E.; Carbonell, F.; Colectivo IoÉ; Funes, J. y Vila, I. (1999). La immigració estrangera a Espanya: Els reptes educatius. Barcelona: Fundació "La Caixa". Col-lecció Estudis Socials, 1.

Alegre, M.A. (2005). Educació i immigració: l'acollida als centres educatius. Barcelona: Mediterrània. Col-lecció Polítiques, 44.

- (2006). «L'acollida i l'escolarització de l'alumnat estranger al sistema educatiu català». En: Bonal, X. (dir.). L'estat de l'educació a Catalunya. Anuari 2005. Barcelona: Mediterrània, 301-322.

- (2008). «Educación e inmigración. ¿¿Un binomio problemático?». Revista de Educación, 345, 61-82.

Alegre, M.A.; Benito, R. y González, I. (2008). «Procesos de segregación y polarización escolar: la incidencia de las políticas de zonificación escolar». Profesorado. Revista de Curriculum y Formación de Profesorado, 12 (2), 27.

Alegre, M.A. (coord.); Collet, J.; González, S. y Benito, R. (2005). Una visió comparada a les politiques d'acollida i escolarització de l'alumnat nouvingut: Els casos de Baviera, la Comunitat Francòfona de Bèlgica, Quebec i Anglaterra. Estudio financiado a través de la convocatoria ARIE 2004 y por la Fundació Jaume Bofill.

Alonso, J. y Sosvilla-Rivero, S. (2006). «Human capital in Spain: An estimate of educational attainment». Documentos de Trabajo 2004-08. Fundación de Estudios de Economía Aplicada (FEDEA). 
Aparicio, R. (2001). «La literatura de investigación sobre los hijos de inmigrantes». Migraciones, 9, 171-182.

- (2007). "The integration of the Second and 1.5 Generations of Moroccan, Dominican and Peruvian origin in Madrid and Barcelona». Journal of Ethnic and Migration Studies, 33, 1169-1193.

Aparicio, R. y Tornos, A. (2006). Hijos de inmigrantes que se hacen adultos: marroquies, dominicanos, peruanos. Madrid: Observatorio Permanente de la Inmigración.

Becker, G. (1964). Human capital: A theoretical and empirical analysis, with special reference to education. Chicago: University of Chicago Press.

Benito, R. y González, I. (2007a). Processos de segregació escolar a Catalunya. Barcelona: Fundació Jaume Bofill.

- (2007b). Les desigualtats en l'accés escolar a Catalunya. Barcelona: Mediterrània. Col-lecció Polítiques, 59.

BorJas, G.J. (1999). Heaven's door: Immigration policy and the American economy. Princeton: Princeton University Press.

Carbonell, F. (1998). "La integració social i la diversitat cultural a l'escola». Educar, 22-23, 197-216.

Carrasco, S. (2003). «La escolarización de los hijos e hijas de inmigrantes y de minorías étnico-culturales». Revista de Educación, 330, 99-136.

- (2008). «Segregació escolar i immigració: repensant plantejaments i alternatives». Revista Nous Horitzons, 190, 31-40.

Carrasco, S.; PÀmies, J.; Ponferrada, M.; Ballestín, B. y Bertran, M. (2009). «Segregación escolar e inmigración en Cataluña: aproximaciones etnográficas». EMIGRA Working Papers, 126 (www.emigra.org.es).

Cebolla, H. (2008). «¿Están los estudiantes de origen inmigrante en desventaja?: Diferencias internacionales e interregionales en España». Panorama Social, 8, 7-111.

Centro de Investigación y Documentación Educativa (CIDE) (2005). La atención al alumnado inmigrante en el sistema educativo en España. Madrid: Ministerio de Educación y Ciencia, 328. Colección Investigación, 168.

Colectivo Ió́ (2002). Immigració, escola i mercat de treball: Una radiografia actualitzada. Fundació "La Caixa". Col.lecció Estudis Socials, 11.

- (2003). "Alumnos y alumnas de origen extranjero». Cuadernos de Pedagogía, 326, 63-68.

Defensor del Pueblo (2003). La escolarización del alumnado de origen inmigrante en España: análisis descriptivo y estudio empirico. Madrid.

Essomba, M.A. (1998). "Què es pot fer des de les entitats juvenils per a la integració dels joves immigrants». Educar, 22-23, 229-235.

- (2002). "Oh, benvinguts, passeu, passeu...». Perspectiva Escolar ("Acolliment i diversitat lingüistica»), 266, 11-19.

- (2003). «Diversidad cultural en la educación infantil». Aula de Infantil, 15, 34-37.

Fernández Anguita, M. (dir.) (2007). Las fronteras de la protección social: Percepciones de la población sobre las presentes opciones y posibles alternativas en materia de protección. Vol. I. Informe del Centro de Análisis Sociales de la Universidad de Salamanca, 324.

Ferrer, F. (dir.) (2008). Les desigualtats educatives a Catalunya (I-II). Barcelona: Fundació Jaume Bofill.

Ferrer, F.; Essomba, M.A. y Bonal, F.X. (2005). «Politica educativa e igualdad de oportunidades». Cuadernos de Pedagogía, 344, 82-87.

Gang, I.N. y Zimmermann, K.F. (2000). «Is the child like parent?: Educational attainment and etnnic origen». Journal of Human Resources, 35 (3), 550-569. 
García Fernández, J.A. (2006). «La investigación sobre educación intercultural en España: Evolución temática, metodológica, necesidades y tendencias futuras». Comunicación presentada en el I Congrés Internacional d'Educació a la Mediterrània. Educació, integració i moviments migratoris. Propostes per al segle XXI. Palma de Mallorca, 11-13 de mayo de 2006.

Garreta, J. (2006). "Ethnic minorities and the Spanish and Catalan educational systems: from exclusion to intercultural education». International Journal of Intercultural Relations, 30, 261-279.

Gramberg, P. (1998). «School Segregation: The Case of Amsterdam». Urban Studies, $35(3), 547-564$.

Massey, D.S. y Denton, N.A. (1988). «The dimensions of residential segregation». Social Forces, 67, 281-315.

Ministerio de Educación y Ciencia. Secretaría General de Educación, Instituto de Evaluación (2006). PISA 2006. Programa para la Evaluación Internacional de Alumnos de la OCDE: informe español.

Pajares, M.; Vilaplana, L. y Baños, M. (2004). Inserción laboral de la población inmigrada en Cataluña. 2004. Barcelona: CERES (Centre d'Estudis i Recerca Sindicals).

Palaudàrias, J.M. (2002). «Escola i immigració estrangera a Catalunya: la integració escolar». Papers: Revista de Sociologia, 66, 199-213.

Portes, A. y Zhou, M. (1993). «The new second generation: segmented assimilation and its variants among post-1965 immigrant youth». Annals of the American Academy of Political and Social Science, 530, 74-98.

Portes, A. y Rumbaut, R. (2003). Legacies: The Story of the Immigrant Second Generation. Berkeley: University of California Press.

Portes, A.; Aparicio, R. y Haller, W. (2009). La segunda generación en Barcelona: un estudio longitudinal. Barcelona: Fundació CIDOB.

Sánchez Hugalde, A. (2007). "Efectos de la inmigración en el rendimiento educativo: el caso español». Ponencia presentada en las XVI Jornadas de la Asociación de Economía de la Educación. Gran Canaria, 12 y 13 de julio de 2007.

SAntamaria, E. (1994). "La improbable interculturalitat de l'escola». Ponencia presentada en el II Congrés Català de Sociologia. Girona, 15 y 16 de abril de 1994.

- (2002). «Inmigración y barbarie: La construcción social y política del inmigrante como amenaza». Papers: Revista de Sociologia, 66.

Schindler, B. (2007). «Living and Learning Separately?: Ethnic Segregation of School Children in Copenhagen». Urban Studies, 44 (7), 1329-1354.

Serra, C. y Palaudàrias, J.M. (2007). "L'alumnat de nacionalitat estrangera en els estudis postobligatoris». En: Larios, M.J. y NadAL, M. (eds.). L'estat de la immigració a Catalunya. Anuari 2006. Barcelona: Mediterrània, 301-334.

Síndic De GReuges (2008). Informe extraordinari: La segregació escolar a Catalunya. Barcelona.

Siqués, C. (2008). Les aules d'acollida d'educació primària a Catalunya: descripció i avaluació dels resultats. Tesi doctoral de la Universitat de Girona dirigida per Ignasi Vila Mendiburu i Santiago Perera Izquierdo, 502.

VAliente, O. (2008). "¿A qué juega la concertada?: La segregación escolar del alumnado inmigrante en Cataluña (2001-2006)». Profesorado: Revista de Currículum y Formación del Professorado, 12 (2).

Zinovyeva, N.; Felgueroso, F. y Vázquez, P. (2009). «Inmigración y resultados educativos en España». Efectos económicos de la inmigración en España: Jornadas sobre inmigración: I Informe FEDEA, 139-178. 